\title{
La Hora de la Fenomenología en la Esquizofrenia
}

\section{Time for Phenomenology in Schizophrenia}

\author{
Marino Pérez-Álvarez \\ Universidad de Oviedo
}

\author{
José M. García-Montes \\ Universidad de Almería
}

\author{
Louis Sass \\ Rutgers University
}

Resumen. Este trabajo expone las aportaciones de la fenomenología en el entendimiento de la esquizofrenia. Se empieza por mostrar que la fenomenología viene a recuperar la tradición psicopatológica, perdida a partir del éxito del DSM-III, así como del establecimiento de la neurobiología como corriente dominante. Sus aportaciones se exponen conforme a tres apartados, relativos a la concepción, evaluación y tratamiento. En cuanto a la concepción, la fenomenología concibe la esquizofrenia como un trastorno de la ipseidad, caracterizado por una conciencia intensificada de aspectos normalmente implícitos o pre-reflexivos, por un sentido disminuido de sí mismo como sujeto de la experiencia y de la acción y por la alteración de la articulación perceptiva con el mundo. La evaluación, cuyo principal método es una entrevista semi-estructurada, trata de poner de relieve las experiencias de primera persona que caracterizan el comienzo de la esquizofrenia, para el caso, la antedicha alteración de la ipseidad. A este respecto, se indagan cinco dimensiones: la autoconciencia e implicación en el mundo, la corporalidad, la corriente de conciencia, la delimitación y permeabilidad del yo y la reorientación existencial. Finalmente, se indican las posibilidades que la fenomenología abre para el tratamiento psicológico. Se señalan la actitud fenomenológica como apertura psicoterapéutica (no es lo mismo plantear la esquizofrenia como alteración de la ipseidad que como enfermedad del cerebro) y la importancia de la hermenéutica y de la narrativa. Así mismo, se tratan de ver en la perspectiva fenomenológica las terapias con base en mindfulness y aceptación y compromiso.

Palabras clave: fenomenología, esquizofrenia, ipseidad, psicoterapia de la esquizofrenia.

\begin{abstract}
This study describes the contributions that phenomenology can make to our to understanding of schizophrenia. It begins by showing that phenomenology is recovering the psychopathological tradition lost after the success of the DSM-III and the establishment of neurobiology as the dominant approach. Its contributions are described in three parts: its conceptualization, evaluation and treatment of schizophrenia. Concerning its conceptualization, phenomenology conceives of schizophrenia as an ipseity disorder, characterized by an intensified awareness of things wich are normally implicit or pre-reflexive, by a diminished sense of oneself as the subject of experience and action, and by alteration of perceptive articulation with the world. The main evaluation method is the semi-structured interview, which attempts to underline the first-person experiences characterizing the beginning of schizophrenia, which is the abovementioned alteration of the ipseity. Five dimensions of this alteration are investigated, self-awareness and involvement in the world, bodiliness, stream of consciousness, delimitation and permeability of the self and existential reorientation. Finally, the possibilities offered by phenomenology for psychological treatment are discussed. Approaching
\end{abstract}

La correspondencia sobre este artículo pueden dirigirla al primer autor a la Facultad de Psicología. Plaza Feijóo, s/n.33003-Oviedo. Spain. E-mail: marino@uniovi.es
Reconocimiento: Este trabajo fue realizado en el marco del Proyecto PSI2009-09453. 
psychotherapy from a phenomenological point of view (approaching to schizophrenia as an alteration of ipseity is not the same as for a brain disease), and the importance of the hermeneutics and narrative are also signaled. Furthermore, therapies based on mindfulness and acceptance and commitment are seen from the perspective of phenomenology.

Key words: phenomenology, schizophrenia, ipseity, hyper-reflexivity, schizophrenia psychotherapy.

\section{Introducción}

A pesar de la ingente cantidad de datos empíricos puestos de relieve por la investigación, una concepción unitaria de la esquizofrenia parece todavía lejos, si es que no cada vez más alejada. Así, por ejemplo, la puesta al día de lo que se supone son "justamente los hechos" (Tandon, Keshavan y Nasrallah, 2008a), lejos de ofrecerse como conocimiento acumulativo, vienen a ser en realidad una acumulación de datos que, se diría, "no hay por dónde cogerlos" con miras a una comprensión y explicación psicopatológica. Después de todo, ni la etiopatogénesis está dilucidada (Tandon, Keshavan y Nasrallah, 2008b) ni, por su parte, los "hechos" neurobiológicos, llamados "biomarcadores", cualifican para el diagnóstico, que sigue dependiendo de criterios clínicos (Keshavan, Tandon, Boutros y Nasrallah, 2008). La investigación empírica revisada no parece orientada por una concepción psicopatológica que partiera del "hecho básico" del fenómeno a estudiar. Sin una concepción psicopatológica de lo que sea la esquizofrenia, difícilmente la acumulación de "hechos" contribuye a un mayor y mejor entendimiento del fenómeno. Si, como dijera Kant, los conceptos sin datos empíricos son cojos, los hechos sin conceptos son ciegos. Tanto más ciegos los hechos en la medida en que no parten del hecho básico o de la "cosa misma" a estudiar.

De acuerdo con la fenomenología, la "cosa misma", el hecho básico o la perturbación fundamental que subyace a los "síntomas" por los que se diagnostica la esquizofrenia es una alteración de la experiencia de sí mismo y del mundo (Sass y Parnas, 2003). En esta perspectiva, la esquizofrenia se ofrece ante todo, como un trastorno del yo o de la persona. Si el comienzo de la esquizofrenia consiste en una crisis del sentido de sí mismo (Nelson, Sass y Skodlar, 2009), la recuperación supone precisamente la recuperación del sentido del yo (Davidson y Strauss, 1992).
La fenomenología es una disciplina especialmente interesada en la descripción y explicación de la estructura de la experiencia (Sass, 2010; Sass y Parnas, 2007). La cuestión para la fenomenología es comprender la experiencia en primera persona en el contexto biográfico del yo y sus circunstancias. La fenomenología clínica, aquí referida tiene su base en la fenomenología europea continental fundada por Edmund Husserl. Así, la fenomenología propiamente dicha se propone captar el significado esencial de la experiencia del mundo concreto vivido. El significado esencial se refiere a la forma organizadora y estructuradora, en este caso, de experiencias anómalas como las dadas en la esquizofrenia. La fenomenología trata de describir la organización significativa de las experiencias, expresiones y conductas de las personas, proponiendo un entendimiento narrativo de los individuos. El entendimiento narrativo pone en relación el escenario de la acción con el de la conciencia para dar sentido a las experiencias y conductas de las personas (Stanghellini, 2010; Stanghellini y Lysaker, 2007). Se trata, pues, de una fenomenología interesada en la inter-relación experiencia-conducta-cultura (Pérez-Álvarez, García-Montes, Cangas y Sass, 2008).

La fenomenología constituye probablemente la principal tradición de la psicopatología, con autores clásicos como Karl Jaspers, Eugène Minkowski, Ludwig Binswanger, Klaus Conrad, Ronald Laing, Wolfgang Blankenburg, entre otros. La fenomenología no es sólo una tradición histórica sino que constituye toda una alternativa a la corriente dominante que, con toda su investigación empírica y descripción nosológica, parece más "ciega" y ofuscada que psicopatológicamente orientada. Si bien la fenomenología quedó prácticamente desaparecida a raíz del éxito del DSM-III, es reivindicada en la actualidad como salida y alternativa a la situación creada (Andreasen, 2007). 
La fenomenología está ofreciendo en los últimos años un renovado entendimiento de la esquizofrenia (Bürgy, 2008; Nelson, Sass, Thompson et al, 2009; Parnas, Sass y Zahavi, 2008; Pérez-Álvarez y García-Montes, 2006; Sass, 1992; 2010; Sass y Parnas, 2003; 2007; Stanghellini, 2004a; 2010). Su mayor innovación está en la combinación de una novedosa descripción de la experiencia con la investigación empírica, incluyendo el desarrollo de instrumentos de evaluación (Davidsen, 2009; Parnas, Moller, Kircher, et al, 2005) y de nuevas perspectivas terapéuticas (Pérez-Álvarez, García-Montes, Vallina-Fernández, Perona-Garcelán y Cuevas-Yust, 2010). La verdad es que la fenomenología es hoy la mayor alternativa a la concepción nosológica y neurobiológica dominantes, sin menoscabo de sus posibles conexiones. En el resto del artículo se va a presentar la concepción de la esquizofrenia de esta renovada fenomenología, así como la evaluación y el tratamiento que corresponde a su línea.

\section{Concepción de la esquizofrenia como trastorno de la experiencia de sí mismo}

De acuerdo con el planteamiento desarrollado por Sass y Parnas (2003; 2007), la esquizofrenia se entiende como un trastorno de la experiencia de sí mismo o ipseidad. La ipseidad se refiere al sentido básico del yo, como centro y núcleo de la propia experiencia. Más específicamente, es la modalidad pre-reflexiva, tácita e implícita de la experiencia de sí mismo. Constituye, por así decir, la infraestructura de la autoconciencia y de la propia estructura del yo o, como también se dice, el "yo básico" o "yo mínimo". Ni que decir tiene, la alteración de la ipseidad afecta a toda la persona. En este sentido, la especificación de la ipseidad se sitúa en la línea de la reivindicación de la persona en la esquizofrenia (García-Montes y Pérez-Álvarez, 2003).

De acuerdo con el modelo fenomenológico, el trastorno del yo en la esquizofrenia ocurre en el nivel más básico de la autoconciencia corporal prereflexiva (ipseidad). La alteración de la ipseidad afecta al núcleo de la experiencia de sí mismo y de esta manera a toda la estructura del yo, incluyendo el yo reflexivo y el narrativo y sus aspectos sociales
(Nelson, Sass, Thopmson, et al, 2009). Como dice una paciente, Clara Kean, lo que subyace detrás de los síntomas es un yo atormentado, una experiencia altamente personal, incambiable e irreemplazable por cualquier tratamiento físico. Lo que más me atemorizaba, continúa Kean, era la sensación de que me había perdido, con un constante sentimiento de que mi yo ya no me pertenecía a mí (Kean, 2009).

Tres aspectos caracterizan la alteración de la ipseidad (Sass y Parnas, 2003; 2007): la hiperreflexividad, el sentido disminuido de sí mismo y la alteración de la conciencia del mundo. La hiperreflexividad se refiere a la autoconciencia intensificada de aspectos de sí mismo que normal y funcionalmente son implícitos, tácitos o pre-reflexivos. El caso es que aspectos pre-reflexivos se objetivan, presentándose como si fueran objetos externos (por ejemplo, experiencias cenestésicas o voces alucinatorias). La hiper-reflexividad aquí no comporta un proceso ni una actitud intelectual sino la presentación a la conciencia de aspectos implícitos que irrumpen en el campo de atención y se hacen así objeto de ocupación y preocupación. Se trata de un tipo de hiper-reflexividad en el que, por así decirlo, el "sistema operativo" pre-reflexivo se torna consciente para uno mismo (Sass y Parnas, 2007). El sentido disminuido de sí mismo se refiere al debilitamiento de la intensidad o vitalidad de la propia auto-presencia subjetiva de uno o conciencia de sí como sujeto de la experiencia. La propia auto-presencia, consistencia y persistencia o sensación de ser uno mismo deviene alterada ("ya no me siento que soy yo"). Esta disminución del sentido de sí mismo no es una mera alteración de la auto-imagen o de la identidad personal, aunque también las implica, sino que afecta al sentido fundamental de sentirse uno mismo sujeto de la experiencia. La alteración de la conciencia del mundo se refiere a la pérdida de la articulación perceptiva y del contacto vital con la realidad, incluyendo los otros. El mundo se ofrece descontextualizado, de modo que las cosas pierden su articulación y contextura dada por hecho conforme al "sentido común". El mundo pierde su familiaridad y uno se siente extraño y perplejo. Los demás parecen des-humanizados y desvitalizados, como si no fueran humanos o lo fueran sólo aparentemente. 
Se puede plantear la cuestión acerca de si es primaria la alteración de la autoconciencia (hiperreflexividad y sentido disminuido de sí mismo) respecto de la subsiguiente alteración de la conciencia del mundo (desarticulación perceptiva y pérdida de contacto vital) o son más probablemente aspectos contemporáneos de la conciencia, según el sujeto y el objeto se constituyen mutuamente y el cuerpo y el mundo co-existen conforme a un balance entre modos tácitos y explícitos de conciencia. De acuerdo con Sass y Parnas (2003; 2007), la hiper-reflexividad, el sentido disminuido de sí mismo y la pérdida de la articulación perceptiva son aspectos del trastorno de la ipseidad que se implican mutuamente. En términos de su experiencia, los pacientes parecen confirmar esta contemporaneidad.

Así, Ely Sacks (2007) pone sus memorias de la esquizofrenia bajo el título "el centro no se puede sostener", referido al yo como centro de experiencia, de manera que la vista, los sonidos, los pensamientos y los sentimientos ya no parecen ir juntos (desarticulación perceptiva). Según dice, el yo se desvanece como un castillo de arena. Por su parte, Clara Kean (2009), refiere una "tensión" entre una poderosa autoconciencia que trata de resistir ante el debilitamiento del yo y su conexión con el mundo. Como dice, mi sentido del yo es totalmente aplastado cuando la "burbuja" que rodea mi autoconciencia es destruida debido a su inestable permeabilidad. Renée en su "diario de una esquizofrénica" (Sechehaye, 1947/2003), hablando de su sentimiento de irrealidad dice: "miraba, por ejemplo, una silla o un jarro, ya no pensaba en su utilidad, en su función: para mi ya no era un jarro que servía para contener agua o leche, o una silla hecha para sentarse. ¡No! ¡Habían perdido su nombre, su función, su significado y se habían convertido en 'cosas!" (p. 138). "Las personas me parecían - continúa Renée - como vistas en un sueño: no distinguía su carácter particular; eran 'humanos' y nada más" (p. 156). La desarticulación perceptiva y pérdida de contacto vital con los otros de Renée implica la desorganización del yo. No en vano Stanghellini (2004a) ha caracterizado la esquizofrenia como crisis global del sentido común.

\section{Evaluación de las experiencias de sí mismo anómalas}

La entrevista es el método por excelencia de la fenomenología. En relación con la esquizofrenia, se dispone de una entrevista semiestructurada específicamente dirigida a la evaluación de la variedad de experiencias que caracterizan la alteración de la ipseidad. Se refiere a la EASE: Examination of Anomalous Subjective Experience (Parnas, Moller, Kircher et al, 2005). La EASE consta de 57 items agrupados en cinco grandes dominios o dimensiones, que son: 1) autoconciencia e implicación en el mundo (presencia), 2) corporalidad, 3) corriente de conciencia, 4) auto-demarcación, y 5) orientación existencial. La identificación de las dimensiones deriva de la investigación empírica, la experiencia clínica y la teoría fenomenológica. La entrevista puede llevar dos sesiones de una o dos horas cada una (Davidsen, 2009). A continuación se van presentar brevemente los dominios señalados de la experiencia anómala, a modo indicativo de los datos o hechos que interesan a la fenomenología.

1) Autoconciencia e implicación en el mundo (presencia) La autoconciencia e implicación en el mundo, nombrada como presencia en la literatura de referencia, se refiere al sentido del yo-como-sujeto de experiencia y a su inmersión en el mundo. La autoconciencia aquí es ante todo autoconciencia pre-reflexiva, implícita, tácita, no temática, que se tiene de sí mismo sin tomarse uno como objeto o tema expreso de atención o reflexión. La autoconciencia pre-reflexiva funciona como condición para la experiencia del mundo y de sí mismo cuando uno es objeto o tema de reflexión (como yo reflexivo). Pero, lo propio de la autoconciencia pre-reflexiva es pasar desapercibida, permaneciendo como "sistema operativo" de la autoconciencia, no como objeto o tema.

En el comienzo de la esquizofrenia, la autoconciencia pre-reflexiva deviene alterada. La alteración consiste en una variedad de cambios cualitativos, tales como el sentido disminuido del yo básico (sensación de vacío interior, sentimientos de anonimato, como si la persona no existiera o fuese profundamente diferente de las demás personas), la distorsión de la perspectiva de primera persona (la sensación 
como si no fuera yo quien está experimentando el mundo), la auto-presencia disminuida ("todo me resulta completamente indiferente"), la desrealización (el mundo circundante parece transformado, irreal y extraño), la hiper-reflexividad ("tuve que pensar acerca de qué pensar"), la pérdida del sentido común (falta de comprensión automática y prerreflexiva del significado de eventos, situaciones, gente y objetos cotidianos).

2) Corporalidad. De la variedad de experiencias alteradas de la corporalidad destaca la tendencia a experimentar el propio cuerpo como objeto, de manera que se hacen explícitos y objetivan aspectos implícitos, normal y funcionalmente desapercibidos. "No soy yo mismo, me siento extraño, ya no estoy en mi cuerpo; siento el cuerpo lejos, en algún lugar. Aquí están mis piernas, mis manos, puedo también sentir mi cabeza, pero no puedo encontrarla de nuevo. Oigo mi voz cuando hablo, pero parece venir de otro lugar", dice un paciente citado por Parnas y Handest (2003). Otro paciente presenta el fenómeno de des-automatización de la acción motora, de manera que actos rutinarios como vestirse o hacer cualquier tarea habitual requieren atención consciente y esfuerzo, teniendo que pensar cada movimiento.

Esta pérdida de contacto vital con el propio cuerpo supone toda una despersonalización que presenta dos formas extremas: la del cuerpo sin alma, de manera que uno vive el cuerpo como si uno fuera un zombi o como algo mecánico, tipo cuerpo-máquina o cyborg, y la del espíritu sin cuerpo, de manera que uno vive como mero espectador de sus percepciones, acciones y pensamientos, como si el cuerpo se hubieran transformado en psico-mecanismo cual scanner (Stanghellini, 2004a). Estas dos formas: cuerpo sin alma (cyborg) y espíritu sin cuerpo (scanner), la segunda apoteosis de la primera, vienen a representar dos formas de experiencia patológica derivadas del dualismo cuerpo-mente (Stanghellini, 2009). Mientras que el cuerpo sin alma (cyborg) representaría una objetivación del cuerpo en detrimento de la mente, el espíritu sin cuerpo (scanner) representaría una objetivación de la mente en detrimento del cuerpo.

3) Corriente de conciencia. Las alteraciones de la corriente de conciencia consisten en algún tipo de fisura entre el yo y sus contenidos, de manera que se interrumpe el normal curso de los pensamientos, de la conciencia y de la vida subjetiva. El fluir de la conciencia deja de ser transparente, descarrila del tema presente y se desquicia su sentido personal. La fisura entre el yo y sus contenidos consiste en la experiencia de los pensamientos como si fueran autónomos, desprovistos de su dimensión subjetiva, descentrados y ajenos a uno mismo, como si vinieran de no se sabe dónde. Así, pensamientos, imaginaciones o recuerdos no pertinentes al tema entre manos, irrumpen en el curso del pensamiento e interfieren con él (interferencia del pensamiento). A menudo son descritos por los pacientes como "pensamientos automáticos" o "tics del pensamiento". A veces pensamientos o imágenes acuden en sucesión rápida dando la sensación de presión en la cabeza ("presión del pensamiento").

Una alteración de la corriente de conciencia característica de este dominio es la espacialización de la experiencia subjetiva, de una manera en la que eventos privados como pensamiento y habla interna se presentan con cualidades físicas, cual objetos densos y localizados en alguna parte de la cabeza. Un paciente citado por Parnas y Handest (2003) describe así esta experiencia: "siento mis pensamientos en la parte derecha del cerebro", "parece como si mis pensamientos estuvieran ligeramente detrás de mí cráneo". Una de las formas prominentes de espacialización de la experiencia es la perceptualización del habla interna o del pensamiento, tradicionalmente reconocida como oír voces o alucinaciones auditivas. El habla interna y el pensamiento normalmente silentes se presentan con cualidades perceptivas auditivas, no necesariamente "captados" por vía auditiva. Así, Stanghellini y Cutting (2003) se refieren a las alucinaciones verbales como "ruptura del silencio del diálogo interno".

4) Auto-demarcación. Las alteraciones de la autodemarcación o delimitación y permeabilidad del yo se refieren al sentido de pertenencia y de autoría de la experiencia, en particular, en qué medida el propio mundo interior es transparente para los otros y los demás lo invaden. La propagación por la que los propios pensamientos son captados por los demás y la inserción por la que los demás ponen sus pensamientos en nosotros son ejemplos de este tipo. 
Los problemas de delimitación entre el yo y el noyo consisten, sobre todo, en la confusión con otro, de manera que el paciente se experimenta a sí mismo y a su interlocutor como estando mezclados o inter-penetrados. Así, pierde el sentido acerca de quién son los pensamientos, los sentimientos o las expresiones, si míos o del otro. El paciente se siente invadido y, a menudo, amenazado por la proximidad corporal de otros. La visión de la propia imagen en un espejo puede dar lugar a un sentimiento de duda acerca de quién es quién y a la evitación de espejos. Las experiencias de ser influenciado por medios técnicos son ejemplos también de alteración de la autodemarcación (Hirjak y Fuchs, 2010).

5) Orientación existencial. Forman parte de este dominio diversas experiencias tipo solipsista, así como el cambio existencial e intelectual. Las diversas experiencias tipo solipsista se refieren, en general, a alguna manera de sentirse único en el mundo, con poderes extraordinarios que pueden incluir la experiencia del mundo como una creación suya (por ejemplo, un producto de su imaginación). Una experiencia solipsista es el fenómeno autoreferencial, donde uno siente una vinculación inmediata entre él y los demás u otros eventos del universo de modo que, por ejemplo, el comportamiento de los demás le parece depender de él (Parnas et al, 2005). Otra experiencia solipsista es la sensación de centralidad, sintiéndose uno el centro del universo.

El cambio existencial o intelectual se refiere a preocupaciones y teorizaciones acerca de temas existenciales, filosóficos, metafísicos, religiosos o psicológicos. Los temas más frecuentes tienen que ver con experiencias místicas, fenómenos para-normales o sobre-naturales, religión, filosofía, psicología, meditación, reencarnación, la vida más allá, la lucha entre dios y el diablo, la comunicación, el sentido de la existencia, el destino de la humanidad e ideas relacionadas con la salud y la nutrición. Estas preocupaciones implican valores que se traducen en actitudes prácticas tales como no sentirse implicado en el mundo más que como mero observador externo (actitud de espectador o de antropólogo). Como dice un paciente citado por Stanghellini y Ballerini (2007), "mi actitud ante la vida puede resumirse del siguiente modo: es como si estuvieran todos en un teatro. Pero mientras todos los demás están pendien- tes de lo que sucede en el escenario yo no puedo dejar de pensar en lo que pasa entre bastidores, en lo que hace la escena posible".

Las dimensiones que distingue EASE se entiende que tienen su "centro de gravedad experiencial" en la ipseidad, con sus tres aspectos señalados: hiperreflexividad, sentido disminuido de sí mismo y alteración de la conciencia del mundo.

\section{Tratamiento de la esquizofrenia fenomenológicamente orientado}

Se podría preguntar “¿qué ha sido de la psicoterapia de la esquizofrenia?" en tiempos de los antipsicóticos. Lo cierto es que después de la década del cerebro y de los grandes avances de la neurociencia, lo principal en relación con el tratamiento de la esquizofrenia está por resolver, si se está de acuerdo en que lo principal es la recuperación del sentido de sí mismo y del protagonismo de la propia vida, más allá del uso eufemístico del término "persona", tomando la persona en serio. Supuesto que el "centro de gravedad experiencial" tiene que ver con aspectos del yo-básico relativos a la autoconciencia pre-reflexiva (ipseidad), una psicoterapia fenomenológicamente orientada iría dirigida a la restauración y fortalecimiento de la experiencia de primera persona y así del sentido del yo. La recuperación del sentido de sí mismo empieza por una actitud fenomenológica abierta a la experiencia y el consiguiente reconocimiento mutuo como personas. A continuación se introduce la posible virtualidad terapéutica de la actitud fenomenológica para, después, hacer referencia a diversas psicoterapias que se pueden ver y explotar en la perspectiva fenomenológica, como las psicoterapias narrativas y con base en mindfulness, aceptación y compromiso.

\section{La actitud fenomenológica como apertura psicoterapéutica}

Por lo pronto, la concepción de la esquizofrenia en términos de la ipseidad proporciona un contexto abierto a la exploración de la experiencia y su sentido, distinto a su concepción en términos del cerebro, donde no habría mucho que hablar con el paciente, 
más allá de preguntar por los síntomas y del trato de cortesía, a veces, como el que "da la razón a un loco". Mientras que la esquizofrenia como enfermedad del cerebro compromete una narrativa de enfermedad donde ya es prácticamente oficial el "modelo de vulnerabilidad", que terminan por aprender los propios pacientes (como si la esquizofrenia fuera una enfermedad crónica más cualquiera, como la diabetes, que con medicación se puede mantener controlada), la esquizofrenia como trastorno de la ipseidad compromete una narrativa de recuperación del sentido del yo, donde se contempla la salida de tal condición (Davidson, 2003).

En términos de la ipseidad, la persona tiene mucho que decir. Para empezar, el papel del clínico no es tanto el de "diagnosticador" como el de participante que escucha y trata de comprender lo que pueden ser experiencias peculiares para el sentido común. No se trata de "adoctrinar" a la persona en la teoría de la ipseidad (como se adoctrina en la de la vulnerabilidad), sino de crear un contexto inter-subjetivo y comprensivo. Sin embargo, todo hay que decirlo, los pacientes se sienten reconocidos en términos de la ipseidad, como muestra la citada Clara Kean (Kean, 2009), cuando reporta su propia experiencia de esquizofrenia en términos de trastorno del yo, tomando como referencia a Sass y Parnas (2003). "Silenciamiento del yo" es lo que Clara había encontrado hasta entonces por parte de la psiquiatría al uso, según titula su reporte. Asimismo, el caso llevado por Nelson y Sass (2009) se reconoce plenamente en la "teoría de la ipseidad", lo que sirvió para "insight" y compromiso con el tratamiento. Ni que decir tiene que la cuestión no está en el empleo de términos técnicos como "ipseidad", sino en la consideración de los modos de experiencia, a menudo silenciados no sólo en la práctica clínica sino en los entornos mundanos.

Sobre esta base, la actitud fenomenológica puede implicar una cierta virtualidad terapéutica, en la medida en que entraña una postura abierta a la experiencia de primera persona y es comprensiva de su sentido, en vez de una postura diagnóstica, observadora de síntomas en tercera persona. La actitud fenomenológica supone atender a las "cosas mismas", que no son otras que las personas ahí, con sus experiencias y modo de ser. La actitud fenomenoló- gica es una actitud de aceptación de la experiencia del otro, toda vez que es su modo real de sentir y experimentar el mundo. El clínico habría de adoptar una suerte de epojé o puesta entre paréntesis de sus propias concepciones y opiniones (otras que no sean las de apertura y aceptación). La epojé supone por parte del clínico la tolerancia a la ambigüedad, sin sentirse obligado a entender todo ni tener que dar una "diagnóstico", explicación o solución.

La esquizofrenia no se vería, sin más, como algo que tiene la persona, según se ha adoptado la fórmula dogmática, por razones de supuesta corrección política, de "persona con esquizofrenia". La esquizofrenia es también un modo de ser, por lo que no habría de desdeñarse la expresión "persona esquizofrénica", entre tanto capta posibles cualidades esquizofrénicas de la persona, de acuerdo con Sass (2007). Mientras que la expresión "persona con esquizofrenia" evoca una concepción de enfermedad estándar, como si ésta estuviera añadida a la persona sin implicaciones idiosincrásicas, la expresión "persona esquizofrénica" es respetuosa con las cualidades esquizofrénicas de la persona, referidas a su peculiar forma de experiencia y modo de ser-en-elmundo. Como dice Sass (2007), la expresión "persona esquizofrénica" sugiere que debemos escuchar tan cuidadosa y empáticamente como sea posible las cualidades esquizofrénicas específicas de la persona, que acaso muestran su particularidad individual y entrañan un punto de vista que puede tanto desafiar como enriquecer el nuestro.

El contexto inter-subjetivo y la comprensión son fundamentales para una posible recuperación o restauración del sentido del yo alterado. La fenomenología considera que la posible recuperación implica el reconocimiento mutuo como personas y la creación de significados compartidos. A este respecto, se hace necesario señalar la inter-sujetividad y la hermenéutica como integrantes de la fenomenología (Sass, 1988; Stanghellini, 2010).

\section{Intersubjetividad, hermenéutica y psicoterapia narrativa}

Una relación terapéutica fundada en la actitud fenomenológica proporciona un contexto donde el 
paciente puede desarrollar una perspectiva de primera persona y una autoconciencia pre-reflexiva más robusta. El reconocimiento mutuo como personas es una condición necesaria para el restablecimiento de la perspectiva de primera persona y la reapropiación de la propia experiencia. La psicoterapia fenomenológica tiene lugar entre el terapeuta y el paciente, no dentro del paciente. Stanghellini y Lysaker (2007) han mostrado que esto ocurre en la sesión terapéutica, cuando el terapeuta ofrece consistentemente el punto de vista de un "tú" dirigido a otro "tú", diferente del punto de vista de tercera persona, hablando por ejemplo de la enfermedad o de algún supuesto mecanismo. La cuestión es restablecer en el aquí-y-ahora y en la relación yo-tú la articulación de la persona con la experiencia presente. La psicoterapia se entiende como "prótesis dialógica" para restablecer la conexión debilitada entre los sentimientos y las situaciones interpersonales (Stanghellini y Lysaker, 2007).

$\mathrm{Si}$ la relación terapéutica crea el contexto intersubjetivo necesario sobre el que restablecer la ipseidad, la hermenéutica contribuye a la creación de narrativas que ayudan a la reconstrucción del sentido del yo. Una narrativa de recuperación puede disponer las cosas diferentemente de una narrativa de enfermedad crónica y tratamiento de por vida. El poder reconstructivo de la narrativa tiene su base en la estructura lingüística de la experiencia y en el propio sentido narrativo de la identidad personal (Sass, 1988). La hermenéutica tiene su mayor papel en la reconstrucción del sentido de la experiencia psicótica, tomando la forma narrativa de una historia personal. No se trata de una interpretación impuesta por el clínico, a título de explicación o modelo psicopatológico, sino de la reconstrucción del sentido de las experiencias en el contexto biográfico, donde el propio paciente es autor (Roe y Davidson, 2005). Se entiende que la reconstrucción del sentido de agencia y de autoría de la historia de uno no es un mero subproducto de la recuperación, sino una dimensión clave de la propia recuperación. Ni que decir tiene que se refiere aquí a la autoría de una historia compartida, con coherencia inter-subjetiva, construida en un contexto hermenéutico, y no la autoría del delirio con el que el paciente da sentido a sus experiencias anómalas.
Aunque la narrativa en la esquizofrenia está siendo cada vez más considerada (Roe y Davidson, 2005; France y Uhlin, 2006), una psicoterapia narrativa en la línea de la ipseidad está por desarrollar. De todos modos, psicoterapias narrativas con distinto compromiso fenomenológico se han ofrecido en los últimos años (Pérez-Álvarez et al, 2010). Entre ellas, cabe citar el modelo cognitivo-interpersonal de Gumley y Schwannauer (2006), el modelo narrativo cognitivo conductual de Rodhes y Jakes (2009) y el modelo dialógico de Lysaker y Lysaker (2008).

\section{Terapias con base en mindfulness, aceptación y compromiso $(A C T)$}

Se trata de dos enfoques, a menudo integrados en uno, cuya característica común es cambiar la relación con las experiencias disconfortantes, en este caso, experiencias psicóticas, en el sentido de dejar de reaccionar a ellas y en su lugar experimentarlas tal como se presentan (mindfulness) o aceptarlas con una actitud abierta (aceptación). Tienen su base en el hecho de que el problema no estaría tanto en la experiencia per se como en determinadas reacciones a ella, en particular, su intento de control o evitación. El caso es que los pacientes se ven a menudo perdidos en ciclos de lucha, tratando en vano de deshacerse de voces, pensamientos, imágenes o sensaciones. Al final, se sienten tiranizados por la psicosis y con poco o, en algunos casos, ningún sentido de agencia. Mientras que mindfulness se queda en la experienciación, la terapia de aceptación y compromiso (ACT) conlleva el compromiso de actuar en la dirección hacia los valores. La tarea terapéutica no es tanto eliminar "síntomas" como cambiar la relación ellos (Pérez-Álvarez, García-Montes, PeronaGarcelán y Vallina-Fernández, 2008).

Mindfulness. Aunque tiene su origen en el Budismo, mindfulness habita hoy por derecho propio en la terapia psicológica, haciéndose un espacio en el mundo de la psicosis, de la mano sobre todo de Paul Chadwick y colaboradores (Abba, Chadwick y Stevenson 2008; Chadwick, 2006; Chadwick, Hughes, Russell, Russell y Dagnan, 2009). El paso de sentirse abrumado a alcanzar una cierta paz liberadora pasa por cambiar la relación con la psicosis. 
Este cambio se ha propuesto que ocurre en tres etapas. La etapa 1 supone el cambio fundamental de descentrarse de las experiencias psicóticas y centrarse en la persona con su conciencia de las voces, pensamientos, etc. Esto se puede lograr anclando la experiencia en la respiración y el cuerpo, concentrándose suavemente en lo que está presente. Los participantes refieren que son capaces de notar la fugacidad de las experiencias, sin tener que estar a expensas de ellas. Su respiración y su cuerpo empiezan a ser un lugar seguro, un remanso de paz. La etapa 2 supone la liberación de los ciclos habituales de lucha con la experiencia psicótica. La persona aprende a separar sensaciones y reacciones y puede ver la fugacidad de las primeras, sin tener que responder necesariamente. La etapa 3 supone la adquisición de poder de uno sobre la propia psicosis, mediante la aceptación.

En la medida en que uno acepta las experiencias perturbadoras como una parte del yo, la psicosis no define ni totaliza el yo. Uno tiene las experiencias que tiene, pero no está tenido por ellas. El yo recupera poder en relación con la psicosis. Los síntomas quizá siguen presentes, según los mide la fenomenología descriptiva, en términos de forma y frecuencia, pero la fenomenología de la experiencia subjetiva y de lo que los síntomas significan y comportan para uno es probablemente bien distinta.

$A C T$. Es una terapia de raigambre conductista que se presenta a sí misma como una nueva generación de la terapia de conducta. Cuenta con una sólida investigación sobre el lenguaje y sus implicaciones clínicas, psicopatológicas y terapéuticas. Entre sus novedades figuran: la definición de la evitación experiencial como una dimensión funcional común a diversos trastornos, la aceptación como principio terapéutico incorporando a menudo mindfulness y la inclusión explícita de los valores como orientación de la vida, alternativa al centramiento en el problema. Así, uno podría emprender acciones y establecer compromisos de hacer algo a pesar de ciertas experiencias (voces, pensamientos, etc.). El término "compromiso" que figura en el nombre de esta terapia y su acrónimo ACT para pronunciar 'act', sugieren este aspecto orientado a la acción y activación de la persona en dirección a valores.

Mindfulness y ACT no se ofrecen como una cura para la psicosis, porque la presencia de síntomas psicó- ticos puede que siga. En vez de ello, sus objetivos son enseñar a los participantes a responder diferentemente a sus experiencias psicóticas y a ayudarles a reemprender el curso de la vida (en particular ACT). Estudios muestran que los participantes aprenden una actitud aceptadora y hasta contemplativa de las voces, pensamientos e imágenes y que ello va asociado a una mejora en el funcionamiento clínico general (Chadwick, 2006; Chadwick et al, 2009). Estudios controlados aplicando ACT muestran su eficacia en reducir la recaída en gente con psicosis (Bach y Hayes, 2002; Gaudiano y Herbert, 2006). Un estudio de caso mostró la combinación de aceptación y compromiso en la reorganización de la vida del paciente (VeigaMartínez, Pérez-Álvarez y García-Montes, 2008).

Mindfulnes y ACT tienen al menos tres aspectos relevantes en una perspectiva fenomenológica. En primer lugar, su énfasis en la conciencia plena y abierta de la experiencia viene a ser una especie de puesta entre paréntesis (epojé) de las reacciones, juicios y prejuicios que espontáneamente suelen tener las personas y que, según se ha dicho, pueden estar en la base del problema. En segundo lugar, suponen la existencia de un yo trascendental como referencia que permanece y transciende las distintas y transitorias experiencias. En tercer lugar, ambas pueden contribuir en atenuar la hiper-reflexividad y en mejorar el sentido de yo. En relación con mindfulness, ACT incluye la inmersión en actividades, no meramente para estar ocupado o distraído, sino tendentes a valores, con el compromiso de actuar en su dirección, a pesar de ciertas experiencias como voces que amenacen o comanden otra cosa.

\section{Conclusiones y direcciones futuras}

La fenomenología ofrece hoy una concepción renovada de la esquizofrenia, con base en la investigación empírica, la experiencia clínica y un novedoso análisis fenomenológico (Sass y Parnas, 2003; 2007). Cuenta así con instrumentos de evaluación cualitativa y cuantitativa como EASE (Parnas et al., 2005). Asimismo, la fenomenología abre una "nueva vida para la psicoterapia de la esquizofrenia" (Pérez-Álvarez et al., 2010). La fenomenología viene a recuperar la tradición de la psicopatología, 
eclipsada por la tendencia nosológica y neurobiológica dominantes en psiquiatría y psicología. La aportación fundamental de la fenomenología es la "vuelta a las cosas mismas" y la recuperación de la perspectiva de "primera-persona", fundamentales en la psicopatología que merezca su nombre.

La investigación neurobiológica seguirá creciendo, cómo no, y nuevas ediciones del DSM aparecerán sin, probablemente, cambios sustantivos en la concepción nosológica, pero la fenomenología también seguirá creciendo, según muestra la tendencia actual. La década de 2010 bien podría ser la década de la fenomenología, aun sin estar declarada como tal. La creciente presencia de la fenomenología puede contribuir a sacar a la terapia psicológica de la metáfora neuroléptica (Birchwood y Trower, 2006), haciendo ver que son otras sus aportaciones, entre ellas la recuperación del sentido del yo.

Con todo, una cuestión todavía pendiente por parte de la fenomenología tiene que ver con la génesis de las alteraciones descritas, una cuestión por lo demás abierta en psiquiatría y psicología clínica. La menor dedicación de la fenomenología a la génesis no sólo es una limitación sino que corre el riesgo de echar a perder sus aportaciones, en la medida en que terminen por verse como epifenómenos de supuestas alteraciones neuroevolutivas. No es que los procesos neuronales no estén presentes, puesto que lo están en todo, incluyendo, valga por caso, los "delirios de los esquizofrénicos" y las "teorías sobre los delirios" de los propios científicos. La explicación que corresponde a la fenomenología es una explicación histórica, acerca de la génesis biográfica de las experiencias anómalas, de acuerdo con las circunstancias personales y el contexto social-cultural. La explicación histórica está en el mismo plano constitutivo, constructivo y genético de los fenómenos a explicar, sin saltar a otro plano causal, por ejemplo, el neuroquímico. Aun existiendo este otro plano, como se suele sostener hoy con neuroimágenes, acaso está más para ser explicado que para ser tomado como explicación. La investigación sobre plasticidad cerebral muestra que la experiencia, la conducta y la cultura modulan el funcionamiento y estructura del cerebro, de manera que el cerebro es tanto o más variable dependiente que independiente o causal (Pérez-Álvarez, 2011). Quiere decir que una explicación histórico-biográfica puede ser más pertinente y potente que la explicación científico-natural, cuando de experiencias y actividades humanas se trata.

Se necesitan estudios de caso comprensivos, que traten de capturar las experiencias y acciones en el contexto de las circunstancias personales y culturales. A este respecto, son importantes estudios que indaguen las raíces de la esquizofrenia en la adolescencia, en una perspectiva evolutiva (Stanghellini, 2004b). Asimismo, son importantes estudios que traten de establecer las mediaciones causales de la cultura sobre la esquizofrenia como el llevado por Sass (1994) a propósito del "caso Schreber".

\section{Extended Summary}

We begin by showing the role of phenomenology in recovering the psychopathological perspective, lost in times dominated by neurobiology and the DSM. While neurobiological research presents a large number of "facts" (Tandon, Keshavan and Nasrallah, 2008) which, not to belittle their importance, say little about understanding schizophrenia as a psychopathological phenomenon, reduced by the DSM to a few symptoms for diagnosis, which dissuades clinicians from knowing their patients as individual persons (Andreasen 2007). On the contrary, phenomenology attempts to describe and explain the structure of the subjective experience, in this case, usually of persons who are diagnosed with schizophrenia (Sass, 2010). According to phenomenology, the basic fact or main perturbation characterizing schizophrenia is an alteration of awareness of oneself and the world (Sass and Parnas, 2003; 2007). From this perspective, schizophrenia is, above all, a disorder of the self or of the person. Phenomenology starts out from the first person experience, as the "thing itself", to describe and explain, different from the third person approach that is usually adopted by neural-psychological theories, which give more attention to impersonal mechanisms than to personal experiences. With all of this, phenomenology is not limited to describing subjective experience, but tries to identify the basic 
structure or essential disturbance which organizes, or rather, disorganizes, the variety of anomalous experiences, in this case, schizophrenia.

On the basis of structural phenomenology, which is becoming relatively different from descriptive phenomenology, a renewed conception of schizophrenia has been developed that includes an evaluation procedure and new psychotherapeutic perspectives.

The current conception of phenomenology, in particular, following Sass and Parnas (2003; 2007), conceives schizophrenia as a disturbed ipseity. Ipseity is the pre-reflexive experience of oneself (bodily or not). Altered ipseity is characterized by three interrelated aspects, hyperreflexivity, diminished self-affection and loss of vital contact with reality. Hyperreflexivity refers to intensified self-awareness of aspects about oneself that are normally implicit, tacit or pre-reflexive, such as, for example, cenestesic experiences or sonorization of thought. Diminished self-affection refers to weakening of self-presence as the subject of experience and action (for example, "I no longer feel that I am myself"). Loss of vital contact with reality means an alteration of familiarity with the world taken for granted (feeling strangeness and perplexity).

The evaluation procedure developed along these lines is the EASE, the Examination of Anomalous Subjective Experience (Parnas et al, 2005). This semi-structured interview explores five domains or dimensions of self-awareness, or in the case of ipseity, presence, corporality, stream of consciousness, self-demarcation, and existential orientation. This procedure is like a clinical specification of the alteration of ipseity. The dimensions distinguished by the EASE are understood to have their "experiential center of gravity" in ipseity, in the three aspects indicated, hyperreflexivity, diminished self-affection and altered awareness of the world.

The new psychotherapeutic perspectives have been specified in three points. The first is opening psychotherapy with a phenomenological attitude. To begin with, schizophrenia as an ipseity disorder suggests a "narrative of recovery" of the sense of self, different from its consideration as a brain disease which, on the contrary, suggests a "narrative of illness", often as a chronic disease at the expense of life-time medication and without the patient having much to say. The patients themselves feel recognized in terms of ipseity, as their experiences and way of life are taken seriously in their own right, and understood according to the phenomenological attitude (Kean, 2009; Nelson and Sass, 2009). The second point is the role of inter-subjectivity, the hermeneutics and narrative for a phenomenologically informed psychotherapy (Stanghellini and Lysaker, 2007). The three point refers to therapies based on mindfulness and acceptance and commitment, as helpful in changing the pathogenic relationship with own experiences (Charwick, Hughes, Russell et al, 2009; Pérez-Álvarez et al, 2008; Veiga-Martínez et al, 2008). The role these therapies have in attenuating hyperreflexivity, in particular, when in addition to mindfulness and acceptance, they include immersion in activities significant to life, grounded in values and not merely as distractions. Psychotherapy could have a new life in the light of phenomenology (Pérez-Álvarez et al, 2010).

Neurobiological research will continue growing, of course, and new editions of the DSM will appear, probably without substantial changes in its nosological conception, but phenomenology will also continue growing, as the current trend shows. The decade of 2010 could well be the decade of phenomenology, even though it is not declared as such. The growing presence of phenomenology could contribute to removing psychological therapy from the neuroleptic metaphor (Birchwood and Trower, 2006), showing that it has other contributions, among them, the recovery of the sense of self.

\section{Referencias}

Abba, N., Chadwick, P. y Stevenson, C. (2008). Responding mindfully to distressing psychosis: a grounded theory analysis. Psychotherapy Research, 18, 77-87.

Andreasen, N. C. (2007). DSM and the death of phenomenology in America: an example of unintended consequences. Schizophrenia Bulletin, 33, 108-112. 
Bach, P. y Hayes, S. C. (2002). The use of Acceptance and Commitment Therapy to prevent the rehospitalization of psychotic patients: A randomized controlled trial. Journal of Consulting and Clinical Psychology, 70, 1129-1139.

Birchwood, M. y Trower, P. (2006). The future of cognitive-behavioural therapy for psychosis: not a quasi-neuroleptic. British Journal of Psychiatry, 88, 107-108.

Bürgy, M. (2008). The concept of psychosis: historical and phenomenological aspects. Schizophrenia Bulletin, 34, 1200-1210.

Chadwick, P. (2006). Persona-based cognitive therapy for distressing psychosis. Chichester: Wiley.

Chadwick, P., Hughes, S., Russell, D., Russel, I. y Dagnan (2009). Mindfulness groups for distressing voices and paranoia: a replication and randomized feasibility trial. Behavioral and Cognitive Psychotherapy, 37, 403-412.

Davidsen, K. A. (2009). Anomalous self-experience in adolescent at risk of psychosis. Psychopathology, 42, 361-369.

Davidson, L. (2003). Living outside mental illness. Qualitative studies of recovery in schizophrenia. Nueva York: The New York University Press.

Davidson, L. y Strauss, J. S. (1992). Sense of self in recovery from severe mental illness. British Journal of Medical Psychology, 65, 131-145.

France, C. M. y Uhlin, B. D. (2006). Narrative as oucome domain in psychosis. Psychology and Psychotherapy: Theory, Research and Practice, 79, 53-67.

García Montes, J. M. y Pérez Alvarez, M. (2003). Reivindicación de la persona en la esquizofrenia. International Journal of Clinical and Health Psychology, 3, 107-122.

Gaudiano, B. A. y Herbert, J. D. (2006). Acute treatment of inpatients with psychotic symptoms using Acceptance and Commitment Therapy: Pilot results. Behaviour Research and Therapy, 44, 415-437.

Gumley, A. y Schwannauer, M. (2006/ 2008). Volver a la normalidad después de un trastorno psicótico. Bilbao: DDB.

Hirjak, D. y Fuchs, T. (2010). Delusions of technical alien control: a phenomenological description of three cases. Psychopathology, 43, 96-103.
Kean, C. (2009). Silencing the self: schizophrenia as a self-disturbance. Schizophrenia Bulletin, 35, 1034-1036.

Keshavan, M. S., Tandon, R., Boutros, N. N. y Nasrallah, H. A. (2008). Schizophrenia, "just the facts": what we know in 2008. Part 3: Neurobiology. Schizophrenia Research, 106, 89-107.

Lysaker, P. y. Lysaker, J. (2008). Schizophrenia and the fate of the self. Oxford, UK: Oxford University Press.

Nelson, B. y Sass, L. A. (2009). Medusas' stare: a case study of working with self-disturbance in the early phase of schizophrenia. Clinical Case Studies, 8, 489-504.

Nelson, B., Sass, L.A. y Skodlar, B. (2009). The phenomenological model of psychotic vulnerability and its possible implications for psychological interventions in the ultra-high risk ('prodromal') population. Psychopathology, 42, 283-292.

Nelson, B., Sass, L. A., Thompson, A., Yung, A. R., Francey, S. M., Amminger, G. P. y McGorry, P. D. (2009). Does disturbance of self underlie social cognition deficits in schizophrenia and other psychotic disorders? Early Intervention in Psychiatry, 3, 83-93.

Parnas, J. y Handest, P. (2003). Phenomenology of anomalous self-experience in early schizophrenia. Comprehensive Psychiatry, 44, 121-134.

Parnas, J., Moller, P., Kircher, T., Thalbitzer, J., Jansson, L., Handest, P. y Zahavi, D. (2005). EASE: Examination of Anomalous Self-Experience. Psychopathology, 38, 236-258.

Parnas, J., Sass, L., y Zahavi, D. (2008). Recent developments in philosophy of psychopathology. Current Opinions in Psychiatry, 21, 578-584.

Pérez-Álvarez, M. (2011). Cerebro, conducta y cultura. Madrid: Alianza Editorial.

Pérez-Álvarez, M. y García-Montes, J. M. (2006). Entendimiento filosófico de la esquizofrenia. Apuntes de Psicología, 24, 11-29.

Pérez-Álvarez, M., García-Montes, J. M., Cangas, A. J., Sass, L. (2008). Defending a phenomenological-behavioral perspective: culture, behavior, and experience. Philosophy, Psychiatry, \& Psychology, 15, 281-285.

Pérez-Álvarez, M., García-Montes, J. M., PeronaGarcelán, S. y Vallina-Fernández, O. (2008). 
Changing relationship with voices: new therapeutic perspectives for treating hallucinations. Clinical Psychology and Psychotherapy, 15, 7585.

Pérez-Álvarez, M., García-Montes, J. M., VallinaFernández, O., Perona-Garcelán, S. y CuavasYust, C. (2010). New life for psychotherapy in the light of phenomenology. Clinical Psychology and Psychotherapy, DOI: 10.1002/cpp.716

Rhodes, J. y Jakes, S. (2009). Narrative CBT for psychosis. New York: Routledge.

Roe, D. y Davidson, L. (2005). Self and narrative in schizophrenia: time to author a new story. Journal Medical Ethics; Medical Humanities, 31, 89-94.

Sacks, E. R. (2007). The centre cannot hold. A memoir of my schizophrenia. Nueva York: Hyperion.

Sass, L A. (1988). Humanism, hermeneutics, and the concept of human subject. En S. B. Messer, L. A. Sass and R. L. Woolfolk (Eds.), Hermeneutics and psychological theory (pp. 222-271). New Brunswick: Rutgers University Press.

Sass, L. A. (1992). Madness and modernism: Insanity in the Light of modern art, literature, and thought. Cambridge, MA: Harvard University Press.

Sass, L. (1994), Civilized madness: schizophrenia, self-consciousness and the modern mind. History of the Human Sciences, 7, 83-120.

Sass, L. A. (2007). ‘Schizophrenic person' or 'person with schizophrenia'? Theory \& Psychology, 17, 395-420.

Sass, L. (2010). Phenomenology as description and as explanation: the case of schizophrenia. En S. Galagher y D. Schmicking, eds., Handbook of phenomenology and cognitive sciences (pp. 635654). Berlin: Springer.

Sass, L. y Parnas, J. (2003). Schizophrenia, consciousness, and the self. Schizophrenia Bulletin, 29, 427-444.

Sass, L. A. y Parnas, J. (2007). Explaining schizophrenia: the relevance of phenomenology. En M. C.
Chung, K. W. M. (Bill) Fulford and G. Graham (Eds.), Recovering schizophrenia (63-95). New York: Oxford University Press.

Sechehaye, M. A. (1947/2003). La realización simbólica y Diario de una esquizofrénica. México: FCE.

Stanghellini, J. (2004a). Disembodied spirits and deanimated bodies. The psychopathology of common sense. Oxford: Oxford University Press.

Stanghellini, G. (2004b). Psychopathological roots of early schizophrenia: adolescent vulnerability, hebephrenia and heboidophrenia. Current Opinion in Psychiatry, 17, 471-477.

Stanghellini, G. (2009). Embodiment and schizophrenia. World Psychiatry, 8, 56-59.

Stanghellini, G. (2010). A hermeneutic framework for psychopathology. Psychopathology, 43, 319326.

Stanghellini, G. y Ballerini, M. (2007). Values in persons with schizophrenia, Schizophrenia Bulletin, 33, 131-141.

Stanghellini, J. y Cutting, J. (2003). Auditory verbal hallucinations - breaking the silence of inner dialogue. Psychopathology, 36, 120-128.

Stanghellini, G. y Lysaker, P. H. (2007). The psychotherapy of schizophrenia through the lens of phenomenology: intersubjectivity and the search for the recovery of first- and second-person awareness. American Journal of Psychotherapy, 61, 163-179.

Tandon, R., Keshavan, M. S. y Nasrallah, H. A. (2008a). Schizophrenia, "just the facts": what we know in 2008. Part 1: Overview. Schizophrenia Research, 100, 4-19.

Tandon, R., Keshavan, M. S. y Nasrallah, H. A. (2008b). Schizophrenia, "just the facts": what we know in 2008. Part 2: Epidemiology and etiology. Schizophrenia Research, 102, 1-18.

Veiga-Martínez, C., Pérez-Álvarez, M. y GarcíaMontes, J. M. (2008). Acceptance and Commitment Therapy applied to treatment of auditory hallucinations. Clinical Case Studies, 7, 118-135.

Manuscrito recibido: 01/09/2010

Revisión recibida: 28/09/2010

Manuscrito aceptado: 04/10/2010 